$22^{\text {nd }}$ International Conference on Harbor, Maritime and Multimodal Logistic Modeling \& Simulation

$17^{\text {th }}$ International Multidisciplinary Modeling \& Simulation Multiconference

\title{
Routing Optimization Software for Electric Vehicles Applied to Charging Stations
}

\author{
Andrea Pastorelli ${ }^{1}$, Michela Longo $^{1, *}$, Fabio Borghetti ${ }^{2}$ and Federica Foiadelli ${ }^{1}$ \\ ${ }^{1}$ Politecnico di Milano, Dept. of Energy, Via La Masa 34, 20156, Milan, Italy \\ ${ }^{2}$ Politecnco di Milano, Dept. of Design, Via Durando 38/A, 20158, Milan, Italy \\ ${ }^{*}$ Corresponding author. Email address: michela.longo@polimi.it
}

\begin{abstract}
Nowadays, in the field of mobility, there is much talk about the concept of "Smart Mobility" and the related increase of the electric vehicle market. The latter, however, the great benefits that can bring, they are experiencing some difficulties in mass diffusion. The aim of this work is to develop and propose a tool that identifies the charging possibilities present along the route by simulating a trip with an electric vehicle in order to evaluate the planning of the charging stations in an area and identify any critical issues. For this reason, this work has on the one hand it is to support the user in planning a trip starting from an origin and destination and on the other it can also be used by public and private subjects (decision makers) to identify the best location of the Charging Stations (CSs) in an area. The proposed analytical model has been automated in a software prototype based on spreadsheet and GIS tools. The case studies have been applied in the Lombardy Region (Italy) in order to verify its validity and consistency.
\end{abstract}

Keywords: Charging Stations; Transportation planning; Electric Vehicles; GIS; Modeling.

\section{Introduction}

Today more than ever before, European cities are facing problems caused by transport and traffic. However, the mobility of people and goods is vital for European cities and makes an important contribution to economic growth, employment and competitiveness. The goal of ensuring mobility and at the same time reducing congestion, accidents and pollution is a challenge common to all big cities. Air and noise pollution are examples of commonly shared problems in European cities due to the use of private car. In Italy there was a high use of cars in 2015: in particular, car journeys represented more than $80 \%$ of the passenger-kilometers travelled (European Commission, 2017).

Considering the growing popularity of Electric Vehicles (EVs), Charging Station Placement has become a topic of great interest. This popularity comes from the great advantages that EVs have if compared to traditional inner-combustion vehicles; in fact, they use electrical energy to power the vehicle, which has a lower cost than fossil fuels. Furthermore, fossil fuels are limited resources, while electricity can be easily converted from other forms of energies and generated from renewable sources of energy, which makes EVs more environment friendly. The consumption of fossil fuels by vehicles has caused serious problems of air pollution, especially in big cities, and switching to electric vehicles would help reducing this problem. This is the main reason why society has made a great effort in researching and developing EVs during the past years. Despite all these advantages, Electric Vehicles are encountering some difficulties in spreading in the car market and being accepted by the majority of drivers. That is mainly due to what is called "Range anxiety". Range anxiety is the fear that a

(C) 2020 The Authors. This article is an open access article distributed under the terms and conditions of the Creative Commons Attribution (CC BY-NC-ND) license (https://creativecommons.org/licenses/by-nc-nd/4.0/). 
vehicle might have insufficient range to reach its final destination (Pierre et al, 2011). Figure 1 shows the market share of new alternative-fuel passenger in Italy where BEV (Battery Electric Vehicle); PHEV (Plug-in Hybrid Electric Vehicle); CNG (Compressed Natural Gas). After some years of positive development, the trend has decreased again considerably (European Commission, 2018).

As a consequence, supporting facilities are a vital element for promoting EVs. Electric vehicle charging stations in particular are an essential need for EV drivers: their distribution and size represent vital points in the accessibility and convenience of EVs (He et al, 2013). There are many elements to consider while constructing charging stations, for instance geographical limitations, economic budgets, and, most importantly, the interaction between the charging stations and the EV drivers. In addition, some aspects relating to transportation planning need to be considered. For example, the traffic flow along a road is an important parameter. In this perspective it might be useful to know the current traffic flows to analyze the existing situation; but it might be interesting to estimate the potential traffic flows from specific origins and destinations by implementing origindestination matrices. In this case it is also necessary to study the socio-economic context of a territory/area considering points of interest (e.g. schools, universities, hospitals, shopping centers, industrial areas, etc.).

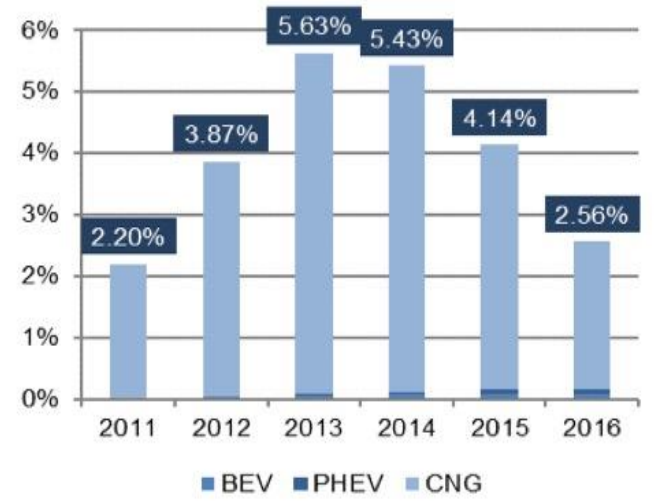

Figure 1. Alternative-fuel passenger cars market share (European Commission, 2017)

These points of interest can be attractors or generators of traffic flows on a transport network (Davidov, 2020). Charging Stations (CSs) are chosen by EV drivers considering their serviceability and position (Zhipeng et al, 2013). Firstly, the serviceability of a CS can be affected by the queues: because of the long time required by the charging process (which, even if shorter than at home, can still take many minutes), queues in charging stations can generate a long waiting time for drivers and ultimately influence the adoption of EVs (Hidrue et al, 2011). Secondly, the position of the charging station is another important issue. Drivers would prefer charging stations located along their path, but if too long a deviation is needed to reach the charging station, the driver could choose not to use that particular station. In return, this would impact on the service quality of the charging station and on the performance of the whole charging system (Xiong et al, 2015). The placement of charging stations is a key research topic in the field of EVs: it is important to make sure that whether drivers need to charge their cars, this can be done in the easiest, fastest and most comfortable way possible (Deilami et al, 2011). Furthermore, the construction of charging stations must be accurately planned, as it is irreversible and requires high expenses. Dynamic pricing schemes can be seen as a flexible complement to charging station placement as they adapt to variances between peak and non-peak time and changes of travel demand. Due to all these aspects, the EVs market is in continuous and fast evolution and therefore some agreements are needed in order to regulate it. The aim of work has on the one hand it is to support the user in planning a trip starting from an origin and destination and on the other it can also be used by public and private subjects (decision makers) to identify the best location of the stations in an area. The proposed analytical model has been implemented in a software prototype based on spreadsheet and GIS tools. The case studies have been applied in the Lombardy Region (Italy) in order to verify its validity and consistency.

\section{Public Charging Infrastructure in Italy}

The charging points can be classified according to the power supplied. They can be of standard power, if they transfer electricity to an electric vehicle with power equal to or less than $22 \mathrm{~kW}$, or of high power, if they allow the transfer of electricity to an electric vehicle with power greater than $22 \mathrm{~kW}$. The high-power charging points divide into two further types: the fast type, if the power is greater than $22 \mathrm{~kW}$ and equal to or less than $50 \mathrm{~kW}$, and the ultra-fast type, if the power is greater than $50 \mathrm{~kW}$.

There are currently 7203 public charging stations in Italy with a total of 13721 charging points. These data consider the number of infrastructures installed up to February 2020, although some are still being connected to the network for activation. The $73 \%$ of the indicated infrastructures are located in a public place (e.g. on the road), while $27 \%$ of them is on private place for public use (e.g. supermarkets or shopping centers). Figure 2 shows the growth between 2019 and 2020 in both the number of charging stations and the number of charging points also distinguishing the percentage of those present in public places (blue) and those in private places (red). It should be noted that the term charging station stands for the infrastructure, while the charging point represents the connection available for a single vehicle (for example, a charging station can have multiple charging points, allowing multiple vehicles to be charged simultaneously). Despite these results, the growth of high-power direct current charges remains weak. In 
fact, the percentage of fast charging points (with power between $44 \mathrm{~kW}$ and $100 \mathrm{~kW}$ ) remains constant at $3 \%$, while the percentage of the ultrafast ones (power over $100 \mathrm{~kW}$ ) is negligible, just 16 in the whole country.

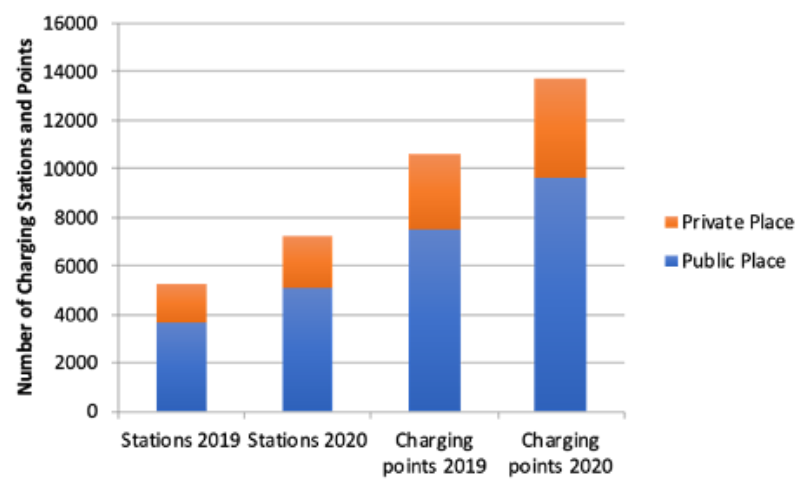

Figure 2. Growth of public stations and charging points between the end of 2019 and the beginning of 2020

\section{Algorithm}

In this case, when the driver has to do a trip using the specific Electric Vehicle, it is important to know the origin point (A) and destination (B) point. The first control is on the State of Charge (SoC) of the EV because in this mode it is possible to determine the total kilometer that it is possible to make. Obviously, if the Soc is equal to $100 \%$ means that the EV could be the maximum kilometers that depends on the vehicle used for the trip.

The EV when starts the path, it calculates the total kilometers that it has to travel to reach the destination (B) and in this way, it is possible to evaluate its remaining range in kilometers (in practical the impact that may have on the State of Charge). If its energy cannot support the whole journey, it will search the closest Charging Station (CS). Another situation could be that the driver could choice a point of interest and make a short stop. In this last case, the vehicle could be recharged.

More specifically, when a driver starts a trip, various possibilities are faced, as reported in Fig. 3.

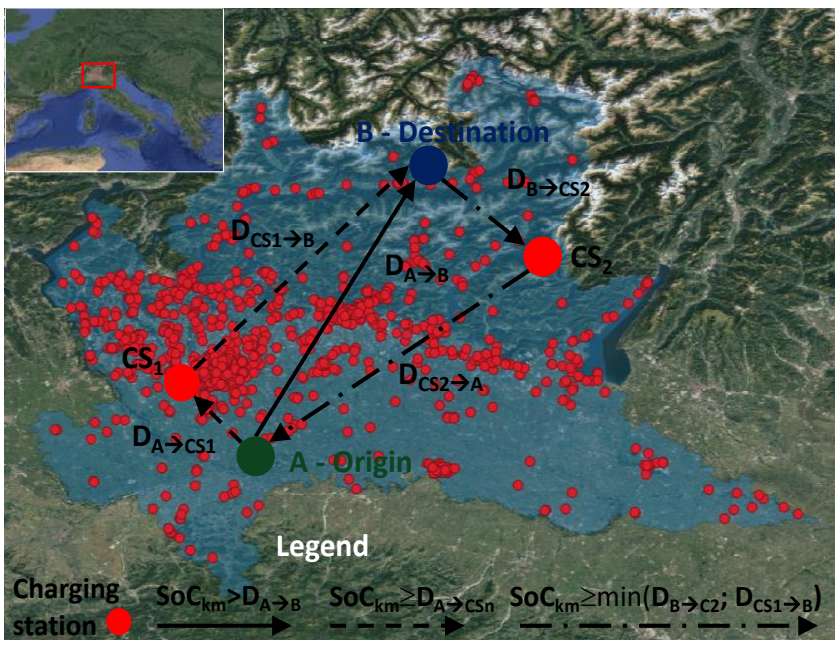

Figure 3. Different possibilities considered in the proposed tool

In particular, it is possible to have different situations:

- The battery level $\left(\mathrm{SoC}_{\mathrm{km}}\right)$ allows reaching the destination directly, in fact in this case:

$$
\mathrm{SOC}_{\mathrm{km}}>\mathrm{D}_{\mathrm{A} \rightarrow \mathrm{B}}+\mathrm{D}_{\mathrm{B} \rightarrow \mathrm{CSn}}
$$

where $\mathrm{CS}_{\mathrm{n}}$ is a general charging station located during the trip and where it is possible to use for the recharge the EV.

- The $\mathrm{SOC}_{\mathrm{km}}$ of the EV is not sufficient to arrive at the destination. For this reason, it is necessary to do a stop in a Charging Station $\left(\mathrm{CS}_{n}\right)$. In this case, it could be this situation:

$$
\mathrm{SOC}_{\mathrm{km}}<\mathrm{D}_{\mathrm{A} \rightarrow \mathrm{B}}+\mathrm{D}_{\mathrm{B} \rightarrow \mathrm{CSn}} \text { AND SOC } \mathrm{km}>\mathrm{D}_{\mathrm{A} \rightarrow \mathrm{CS} 1}
$$

where CS1 is a first charging station closer to the starting point. A good idea is to create a new path for Electric Vehicle, that is:

$$
\mathrm{A}-\mathrm{ORIGIN}_{\rightarrow} \mathrm{CS}_{\mathrm{n}} \rightarrow \mathrm{B}-\text { DESTINATION }
$$

Depending on the kilometers available, or points of interest, the driver can decide whether to prefer a charging station closer to the origin or use a charging station closer to the destination.

- The critical situation is if the $\mathrm{SoC}_{\mathrm{km}}$ is incapable of reaching the destination nor the closest charging station. In this case, the charging station network should be made denser, and another charging station should be inserted in that area

\section{Travel Simulator Tool}

Since the distribution of charging infrastructures is not uniform neither at a national, nor at a local level, it is useful to identify which are the most supplied areas and which are still in backward conditions. Since the final goal is to encourage the use of electric vehicles by putting the driver in the most optimal and comfortable conditions possible, it is also good to perform an analysis from the driver's point of view. Obviously, a driver who travels regularly in areas without recharging points for electric vehicles will be 
unwilling to undertake the use of an EV; vice versa, those who have the opportunity to find easily accessible recharging points along the daily routes and therefore do not waste too much time will be much more motivated to accept the novelty of the EV. This proposed model works by simulating a trip: the user has to enter the coordinates of the starting and destination points, provide the percentage of remaining battery and select a vehicle model among more than 50 possibilities; as a result, the program shows all the possible charging stations located nearby.

\subsection{User Interface}

"User Interface" page, shown in Figure 4, has been included in the tool.

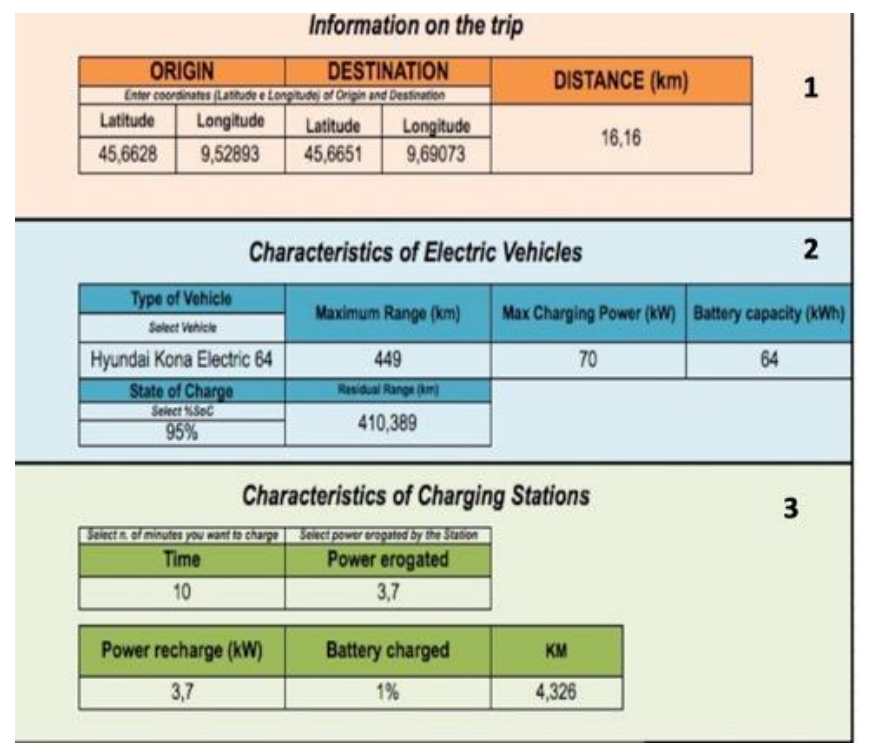

Figure 4. User interface of the tool: (1) Information on the trip, (2) Characteristics of EV and (3) Characteristics of CSs

This page allows the user to enter the required data to carry out the various simulations and allows him to view some outputs. This page is divided into three sections:

1) The first is related to the trip information (Fig. 4(1)): This section is dedicated to the information on the trip, allows the user to manually enter the geographical coordinates (Latitude and Longitude, expressed in degrees) of the Origin (A) and Destination (B) points, subsequently showing the total distance (in $\mathrm{km}$ ) between the two points $\left(D_{A \rightarrow B}\right)$.

2) The second is dedicated to the choice and related information of the vehicle to be used in the simulation (Fig. 4(2)): dedicated to the characteristics of EVs, allows the user to manually choose the vehicle to use for the simulation. By selecting the corresponding box, a drop-down menu containing 53 possible models opens. Each vehicle corresponds to a maximum range of autonomy (in $\mathrm{km}$ ), a maximum charging power (in
$\mathrm{kW}$ ), and a battery capacity (in $\mathrm{kWh}$ ), which are the ones declared by the manufacturers. This information is contained in another page that acts as a database regarding the vehicles, so that it is possible to insert other vehicles with the corresponding information if necessary. It should be specified that as maximum charging power, if the vehicle allows a high-power DC charge, the value indicated is the one corresponding to this one. Once the model has been selected, the State of Charge (SoC) in percentage value that is had at the start must be chosen through another drop-down menu. The program consequently calculates the value of the residual range (i.e. the range that will be passable with the residual SoC, or that which will remain after the trip entered in the first section). In case of too low SoC values (even if it is a very borderline situation), which would not allow to reach the set destination, the message "Charge Needed!"

3) The third deals with the information of the charging stations and the charging times (Fig. 4(3)) dedicated to the characteristics of the charging stations, allows the user to select, through two other drop-down menus, both the time in minutes, which he is willing to use for charging the vehicle, and the power supplied by the identified charging station. Once these two values have been set, the system will show the power to which the charging will take place, it will also show the percentage of rechargeable battery in the selected time interval and the corresponding number of kilometers that can be covered with this recharge. There is also a graph which, based on the power at which the recharge occurs, shows the necessary time (on the abscissa) to recharge a certain percentage of the battery.

\section{Simulation, Analysis and Discussion of Results}

The developed tool, presented in the previous section, has been tested and operated in order to perform various simulations aimed at assessing the presence of charging stations along some routes within the Lombardy Region (Italy), and in order to identify both optimal areas and critical areas.

The simulations presented in this section have been performed using actually about 50 vehicle models (i.e. Nissan Leaf, BMWi3...) with the possibility to extend this set ; each vehicle has been considered take into account different ranges of SoCs percentage with step of $5 \%$ in order to compare the two case study (A and $\mathrm{B}$ ) analysed in this work.

These results are based on two main driver behavioural scenarios. In the first scenario (Case A) the driver opts to stop to recharge its vehicle before reaching the destination; the total route in this case will be Origin - Charging Station - Destination. In the second scenario (Case B), the driver opts, if it is 
possible to do so thanks to the autonomy of the battery, to recharge the vehicle after reaching the destination and carrying out his commitments. In this case the overall route will be Origin - Destination Charging Station. The two case studies were analysed in the Lombardy Region between the provinces of Milano and Bergamo. For the analysis GIS Geographic Information System tools have been used to process data and information and above all to represent the outputs in cartographic form. Figure 5 shows the location and the routes of the two case studies. The red points represent the charging stations and the orange lines indicate the two routes. The following are the two case studies.

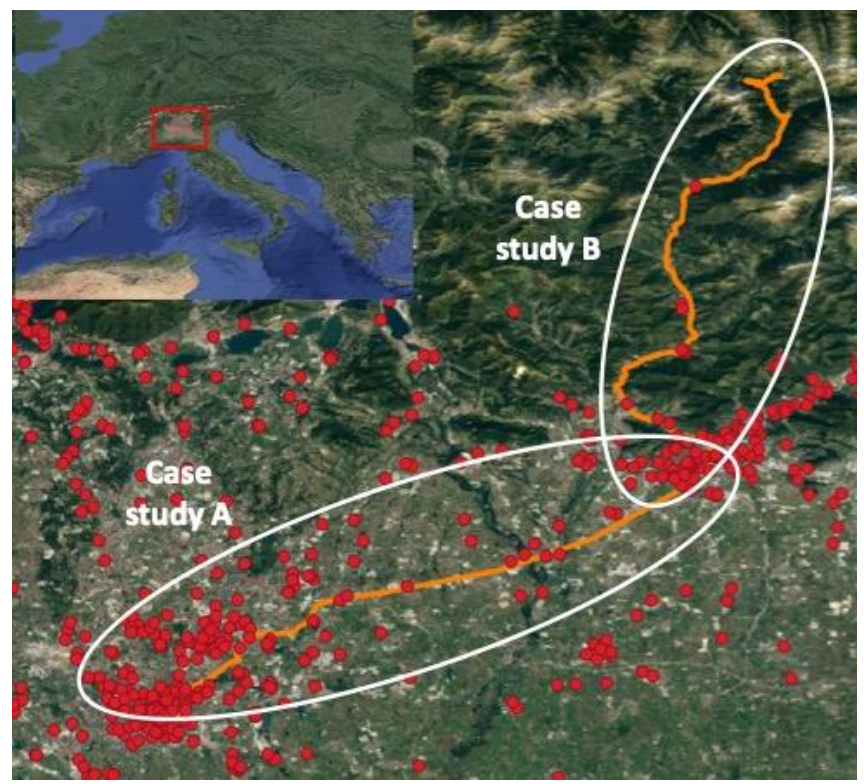

Figure 5. Location and paths of the two case studies

\subsection{Case Study A}

The first simulation was carried out considering Bergamo as the starting point and Milano city as the destination, therefore taking into consideration a longer route: the distance between origin and destination is about $60 \mathrm{~km}$. In this case, the route is mainly on the highway; the overall route will be Origin - Charging Station - Destination. In Figure 6, it is represented the path between the Origin - A (Bergamo) and the Destination - B (Milano).

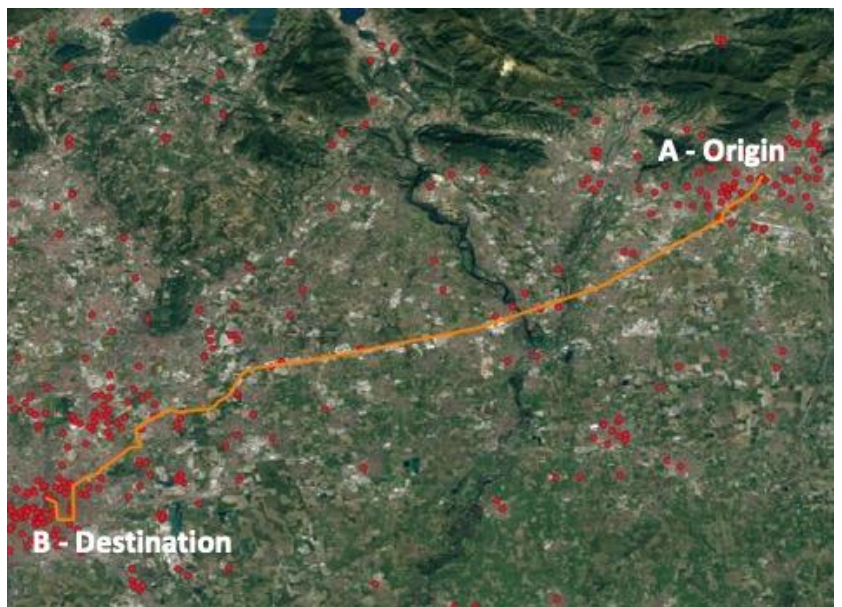

Figure 6. Case study A. Path between the A - Origin and the B Destination

The tool allows the user to choose the distance between the location of the charging stations and the route to reach the destination. Figure 7 shows two buffers: the smaller blue buffer represents a situation where the user is willing to deviate a little from the route; the red buffer represents a greater propensity of the user to deviate a route. By selecting a buffer width (distance $d_{1}$ or $d_{2}$ ) it is possible to identify the charging stations that can potentially be used.

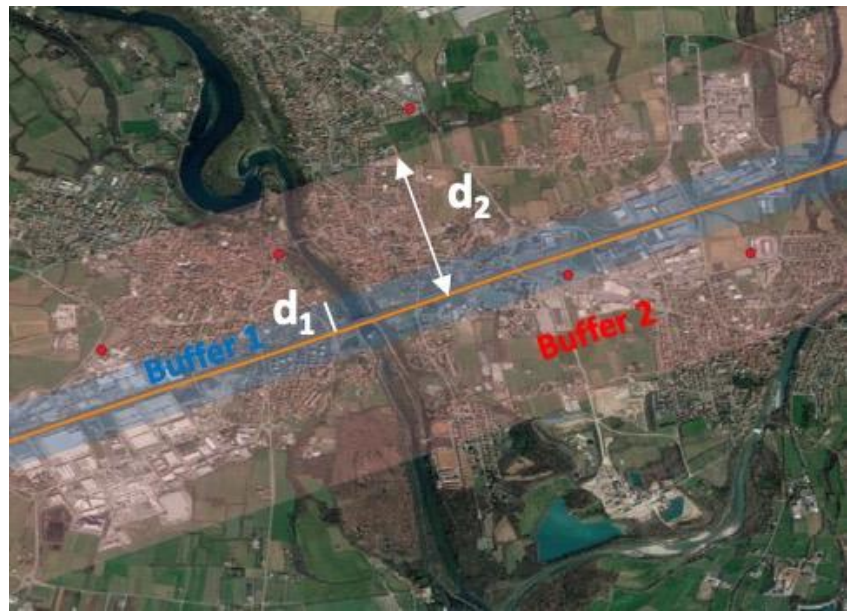

Figure 7. Buffers that allow the user to identify charging stations along the path

When the user has to make a detour to reach the charging station, the model is able to calculate the fastest route. Figure 8 indicates the route deviation from the first available junction along the path and the charging station. 


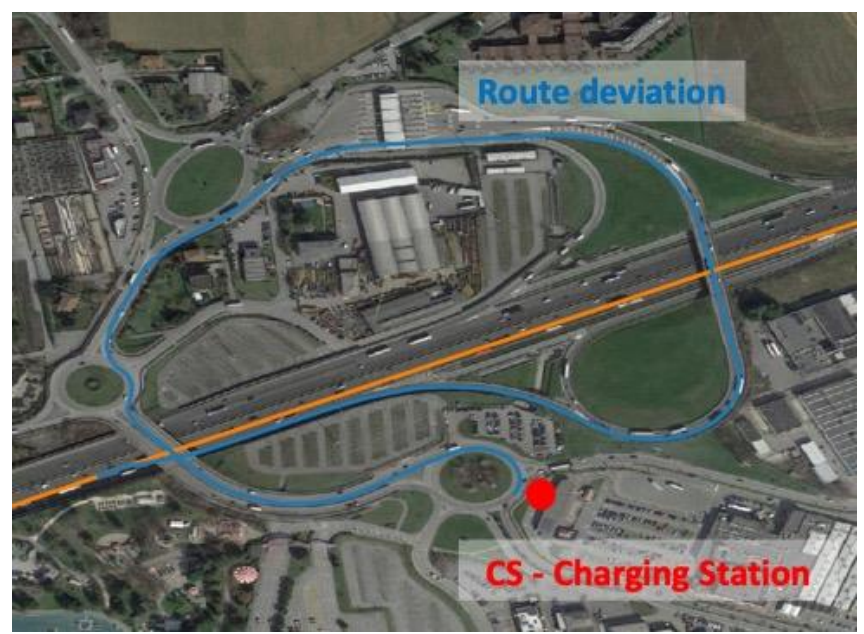

Figure 8. Route deviation from the first available junction along the path and the charging station

Despite this, in the scenarios where it is possible, there is a good number of reachable charging stations that are located near the destination (67) and this means that the center of Milan offers many possibilities to recharge electric vehicles. Further narrowing the research field, it appears that there are sufficient Charging Stations within $1 \mathrm{~km}$ near the Destination. Another consideration is that 110 charging stations emerge along the trip, but only a few of them are reachable in case of limited autonomy. In fact, it emerges that they are located towards the city of Milan and the number of stations decreases moving away from the city, as can be seen from the map (see Fig. 6). It is also possible to note that there is a low number of charging infrastructures along the highway.

\subsection{Case Study B}

In the second simulation, a different situation was considered, with a destination located in a mountain resort. In order to assess whether areas far from city centres were easily accessible to EVs, the town of Foppolo, a ski destination in the province of Bergamo, was taken as a destination. The starting point considered is Bergamo: the distance between origin and destination is about $60 \mathrm{~km}$. In this case the overall route will be Origin - Destination - Charging Station. In fact, unlike case study $A$, there are not enough Charging Stations along the route. In Figure 9, it is represented the path between the Origin - A (Bergamo) and the Destination - B (Foppolo). In this case, the destination could be a critical situation for the recharge of vehicle due to the reduced CSs number; in this way the driver has to make a stop along the route in order to avoid empty battery of EV.

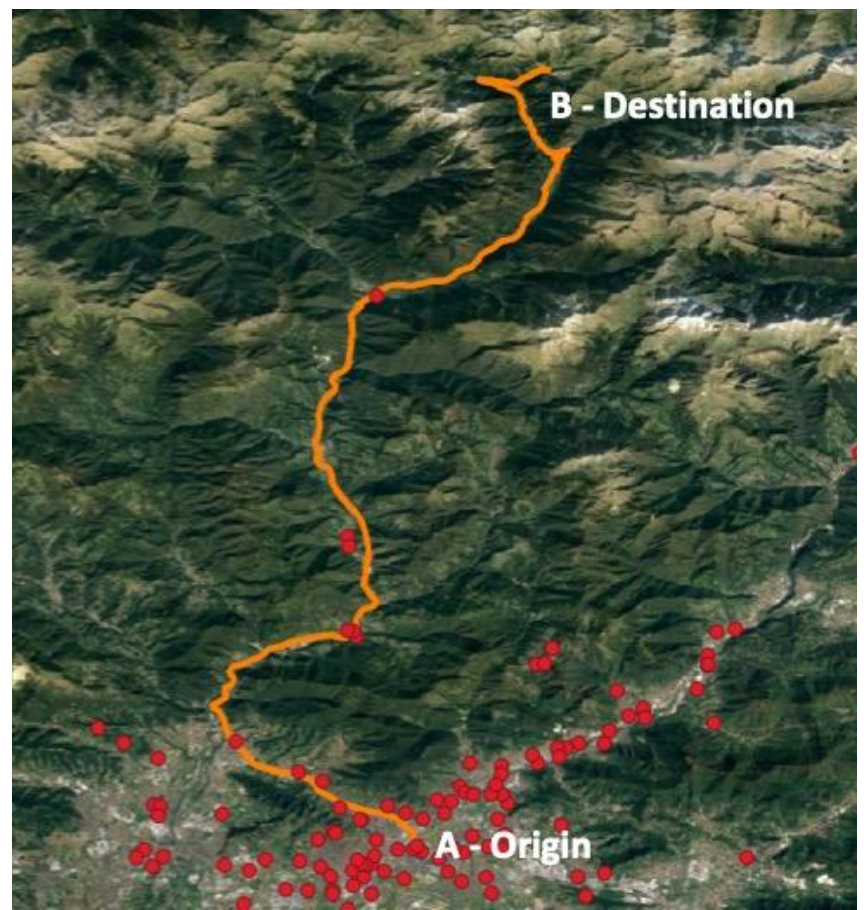

Figure 9. Case study B. Path between the A - Origin and the B Destination

The first fact that the simulation highlights is the complete absence of charging stations within a radius of $5 \mathrm{~km}$ from the destination. The nearest infrastructure for charging electric vehicles is $17 \mathrm{~km}$ away. This is a very negative result, especially considering the fact that the ski resorts in Foppolo are very popular during the winter season and skiers who possess electric vehicles would find it quite difficult to recharge their vehicle. Another consideration, it can be seen that, as one moves away from the origin and enters in the mountain location, the density of recharging stations decreases, thus indicating that in the whole valley there is a real lack of infrastructure for electric vehicles (just 6 stations in $34 \mathrm{~km}$ ). In conclusion, it emerges from the simulation that although the province of Bergamo has a good number of charging stations, it is quite lacking in some particular areas, such as in the mountain zones, which being a fairly frequented holiday resort in certain seasons, would need a marked improvement in terms of number of charging infrastructures.

The simulations confirmed the fact of how the Lombardy Region, and in particular the area between the Provinces of Milan and Bergamo, is at the forefront as regards the planning of charging stations. This situation certainly offers several convenient possibilities for electric vehicle drivers. However, some critical areas emerge, mainly in mountain areas where, there is still a lot of work to be done, if the electric vehicle market is to be spread uniformly, and in the future to replace internal combustion vehicles the as soon as possible. Considering, for example, the aforementioned case of the mountain location, in an optimal scenario it would be advisable to have some 
charging stations near the ski resorts and at least 2 or 3 more charging stations located along the route.

\section{Conclusions}

The conclusions section can end with a paragraph that clearly states the main conclusions of the research along with limitations and directions for future work. Summary illustrations can be included. The main goal of this work is to develop a method of evaluation for the planning of charging stations in a specific area, taking the point of view of a hypothetical electric vehicle driver. Indeed, in an international scenario where the aim is to promote the smart mobility, it is extremely important to analyze the drivers' point of view.

In fact, to encourage mass adoption, it is necessary to act on the that. In this regard, the software it is been developed can be a very useful tool since it makes it possible to simulate trips in different conditions with several types of vehicles and on scenarios. Through the simulations carried out, this software has made it possible to evaluate the planning of charging stations for example in Lombardy Region in Italy and to draw conclusions from them. Apart from this, it can be used in many other situations and applications: it can be applied to other geographical areas and by inserting the charging stations located on the territory; it allows users to enter/add other types of vehicles on which to perform simulations; finally, it also allows to make a study on the effectiveness of a single station by evaluating its charging times. In general, the results obtained from the simulations highlight the spreading of electric mobility are consistent with progress made at European and global level. Nevertheless, they also made it possible to identify some areas where improvements should be made, such as mountain areas, or centers of great importance (commercial centres, school, university, industrial areas, etc). The tool is in fact a DSS - Decision Support System and could be used by different public and/or private subjects to facilitate the programming and planning process related to the location of the charging stations. Depending on specific features of the territory, for example points of interest, it is possible to evaluate the opportunity to charging stations. Overall, however, given the growth trend of power stations in recent years, a scenario in the near future is undoubtedly bright for the spread of electric vehicles.

In the future work, an important factor that will be evaluated by the simulator is waiting time in a station considering the available charging points.

\section{Acknowledgements}

Some information as the territorial distribution of the charging stations (the specific location) and the operators that manage these charging stations present in the Italian territory were provided by the Motus-E.

\section{References}

European Commission. (2018). Transport in the European Union - Current Trends and Issues. https://ec.europa.eu/transport/themes/infrastruct ure/news/2019-03-13-transport-europeanunion-current-trends-and-issues_en

European Commission. (2017). European Urban Mobility - Policy Context. Publications Office of the European Union, 2017, Luxembourg 978-92-7957527-3.

https://ec.europa.eu/transport/sites/transport/file s/cycling-guidance/european_urban_mobility__policy_context.pdf

Davidov, S. (2020). Optimal charging infrastructure planning based on a charging convenience buffer. Energy, 192, 1-10.

Deilami, S.; Masoum, A.S.; Moses, P.S. and Masoum, M.A.S. (2011). Realtime coordination of plug-in electric vehicle charging in smart grids to minimize power losses and improve voltage profile. IEEE Transactions on Smart Grid, 2(3), 456-467.

Funke, S.; Nusser, A. and Storandt, S. (2015). Placement of loading stations or electric vehicles: No detours necessary! Journal of Artificial Intelligence Research, 53, 633-658.

He, F.; Wu, D.; Yin, Y. and Guan, Y. (2013). Optimal deployment of public charging stations for plug-in hybrid electric vehicles. Transportation Research Part B: Methodological, 47(C), 87-101.

Hidrue, M. K.; Parsons, G. R.; Kempton, W. and Gardner, M. P. (2011). Willingness to pay for electric vehicles and their attributes. Resource and Energy Economics, 33(3), 686-705.

Pierre, M.; Jemelin, C. and Louvet, N. (2011). Driving an electric vehicle. A sociological analysis on pioneer users. Energy Efficiency, 4, 511-522

Xiong, Y.; Gan, J.; An, B.; Miao, C. and Bazzan, A.L.C. (2015). Optimal electric vehicle charging station placement. IJCAI'15: Proceedings of the 24th International Conference on Artificial Intelligence, 2662-2668.

Zhipeng, L.; Fushuan, W. and Ledwich, G. (2013). Optimal planning of electric vehicle charging stations in distribution systems. IEEE Transactions on Power Delivery, 28(1), 102-110. 